\title{
Análisis del comportamiento de mercado en las ferias del agricultor de la Gran Área Metropolitana
}

Analysis of market behavior in Farmer's Markets located in the Greater Metropolitan Area

Manuel Enrique García-Barquero ${ }^{1}$

Fecha de recepción: 11 de junio del 2015 Fecha de aprobación: 17 de setiembre del 2015

García-Barquero, M. Análisis del comportamiento de mercado en las ferias del agricultor de la Gran Área Metropolitana. Tecnología en Marcha. Vol. 29, № 1, Enero-Marzo. Pág 83-95. 


\title{
Palabras clave
}

Oferta; demanda; mercado; precios; productos; consumo.

\section{Resumen}

En este artículo se presentan los resultados de un estudio que analizó el comportamiento del mercado de las ferias del agricultor en la Gran Área Metropolitana. Se toman en cuenta las regulaciones de ley para estas actividades y la teoría de la mercadotecnia, con el fin de determinar la forma en que se desarrollan estos mercados a través de las variables de participación de oferentes, clientela habitual por oferente y clientela habitual por producto. Considerando estas variables, se pudo determinar la forma en que los agricultores planifican la oferta de productos agrícolas, el $80 \%$ de los participantes analizados venden entre $84 \%$ y $100 \%$ de la oferta semanal. Es importante mencionar que para el análisis de los productos agrícolas se utilizaron los 20 principales productos hortifrutícolas de mayor comercialización en el mercado mayorista del Centro Nacional de Abastecimiento y Distribución de Alimentos (CENADA); de estos, 18 mantienen una participación constante que varía entre el 50\% y el 83\% durante el año, mientras que el mango y la sandía mantienen un porcentaje menor debido a la estacionalidad de la producción.

\section{Keywords}

Offer; demand; market; prices; products; consume.

\begin{abstract}
A study was conducted in order to analyze the market behavior of farmer's markets in the Greater Metropolitan Area, taking into account the legal regulations of these markets and marketing theory to determine the way in which these markets develop, through the variables of supplier participation, regular clients per supplier and regular clients per product. Considering these variables, the way in which farmers plan the supply of agricultural products was determined: $80 \%$ of participants sell between 84 and $100 \%$ of weakly supply. It is important to mention that for the analysis of this study, the 20 main horticultural and fruit products with higher volume of commercialization in the National Center for Food Supply and Distribution (CENADA) were used. Of these, 18 products held a constant participation, which varies between 50 and $83 \%$ in a year, however, mangoes and watermelons held a lower percentage due to production seasonality.
\end{abstract}

\section{Introducción}

En Costa Rica existen diferentes componentes relacionados con las tendencias de producción, la comercialización y los precios de los bienes agroalimentarios. Estos se ven influenciados por las negociaciones realizadas en el principal mercado mayorista de bienes agroalimentarios, el Centro Nacional de Abastecimiento y Distribución de Alimentos (CENADA), y en las principales ferias del agricultor del Valle Central. Según el artículo “¿Qué es una feria?”, del Consejo Nacional de Producción (CNP), una feria del agricultor es un mercado minorista para uso exclusivo de pequeños y medianos productores, en forma individual u organizada, de los sectores de la producción agropecuaria, pesquera, avícola y pequeña industria y artesanía, en donde venden directamente sus productos al consumidor (CNP, 2014b). 
Cada uno de estos mercados, tanto el mayorista como el minorista, presentan una serie de comportamientos que responden muchas veces a la disponibilidad de productos a lo largo del año, al comportamiento de los consumidores, donde algunas veces los aspectos socioculturales impactan las tendencias de consumo, los precios de los bienes, las condiciones agroclimáticas cambiantes y, finalmente, el comportamiento que tengan las exportaciones e importaciones de productos agroalimentarios.

Las ferias del agricultor son mercados minoristas que impactan directamente al consumidor costarricense, porque han buscado un enfrentamiento cara a cara del productor con los consumidores, proceso en el que la intermediación se reduce a la menor cantidad posible o incluso a su ausencia total (CNP, 2008).

En el análisis del comportamiento de mercado de las ferias del agricultor de la GAM durante 2013 y 2014, el objetivo es poner a disposición de los costarricenses un diagnóstico sobre aspectos o cuestionamientos relacionados con su funcionamiento, poniendo énfasis en las interrogantes sobre la política para poder participar en ellas, dado que la dinámica de comercialización ha sido criticada debido a la participación de intermediarios como oferentes de productos.

\section{Materiales y métodos}

Desde el año 2010, el Centro de Investigación en Economía Agrícola y Desarrollo Agroempresarial (CIEDA) viene desarrollando el proyecto de investigación número 822-B0-119, denominado "Análisis del comportamiento de productos agroalimentarios en el mercado mayorista del CENADA y en las principales ferias del agricultor del Valle Central de Costa Rica". Este proyecto involucra diferentes unidades de investigación como el mercado de mayoristas y las diferentes ferias del agricultor de la GAM, distribuidas, según datos del CNP, en las siguientes subdivisiones: Central-Central, Central-Occidental, Occidental del Este y Occidental del Oeste.

Inicialmente se identificaron las ferias del agricultor ubicadas dentro de la GAM, que fue delimitada por el Plan GAM del Instituto Nacional de Vivienda y Urbanismo (INVU) en 1982, mediante el Decreto Ejecutivo 13583. La GAM está conformada por tres municipios de Alajuela, seis de Cartago, nueve de Heredia y 13 de San José; un total de 31 cantones, que incluyen parcial o totalmente 152 distritos (MIVAH, 2011).

\section{Muestreo}

Para seleccionar las ferias, se consideró el artículo "Entes administradores y emisores de carné de ferias del agricultor" del CNP y se partió de un muestreo estratificado según la subdivisión por región geográfica de la GAM, tal y como se muestra en el cuadro 1. Como se observa, la mayor concentración de ferias se encuentra en las subdivisiones Central-Oriental y CentralCentral (CNP, 2014a).

Considerando lo anterior, se selecciona una muestra de 14 ferias, con base en la precisión que se desea en el estudio y ajustado a las posibilidades de tiempo y financieras. En el cuadro 2 se muestra la distribución por fijación proporcional en cada uno de los estratos previamente definidos, siendo el de mayor participación el Central-Oriental, con seis ferias del agricultor. 
Cuadro 1. Distribución y peso relativo de las ferias del agricultor según la subdivisión por región geográfica de la GAM.

\begin{tabular}{|c|c|c|}
\hline Subdivisión & Número ferias & Porcentaje \\
\hline Central-Oriental & 17 & 38 \\
\hline Central-Central & 13 & 29 \\
\hline Occidental del oeste & 7 & 16 \\
\hline Occidental del este & 8 & 18 \\
\hline Total & 45 & 100 \\
\hline
\end{tabular}

Fuente: Elaboración propia con datos de campo.

Cuadro 2. Número de ferias del agricultor que se analizan en cada una de las subdivisiones geográficas de la GAM.

\begin{tabular}{|c|c|}
\hline Subdivisión & Número de ferias \\
\hline Central-Oriental & 6 \\
\hline Central-Central & 4 \\
\hline Occidental del oeste & 2 \\
\hline Occidental del este & 2 \\
\hline Total & 14 \\
\hline
\end{tabular}

Fuente: Elaboración propia con datos de campo.

Elección de las ferias agricultor

Las ferias del agricultor seleccionadas se eligieron con base en la cantidad de oferentes presentes en ellas, tomando de cada subdivisión las ferias que presentaron la mayor cantidad de puestos ocupados, y que se muestran en el cuadro 3.

Cuadro 3. Ferias del agricultor seleccionadas para el estudio, de acuerdo con la región en que se ubican.

\begin{tabular}{|c|c|}
\hline \multicolumn{2}{|c|}{ Ferias del agricultor, Región Central-Oriental } \\
\hline 1. Zapote & 2. Plaza Víquez \\
\hline 3. Desamparados & 4. Vásquez de Coronado \\
\hline 5. Oreamuno & 6. Tres Ríos \\
\hline Ferias del agricultor, Región Central-Central \\
\hline 1. Hatillo & 2. Pavas \\
\hline 3. Escazú & 4. Alajuelita \\
\hline Ferias del agricultor, Región Occidental del Oeste \\
\hline 1. San Ramón & 2. Grecia \\
\hline Ferias del agricultor, Región Occidental del Este \\
\hline 1. Heredia & 2. Tibás \\
\hline
\end{tabular}

Fuente: Elaboración propia. 


\section{Análisis de los datos}

Mediante un análisis de varianza (ANOVA) y la aplicación de pruebas de diferencia mínima significativa (LSD, por sus siglas en inglés), se identificaron las diferencias que pueden existir entre variables de interés. Todas las pruebas se trabajaron al 5\% de significancia.

Periodo en el que se realizó el trabajo de campo

Como instrumento para recolectar la información, se utilizó una encuesta personal a los propietarios de tramos que participan en las ferias del agricultor seleccionadas. La respectiva validación del instrumento se realizó mediante la visita a una feria donde se aplicó la encuesta. En este sentido, el instrumento se utilizó con diez productores, se recopiló la información, se codificaron los datos y, dado que el proceso no arrojó ningún aspecto controversial, se procedió a realizar el levantamiento de la información.

El objetivo de la encuesta fue cuantificar la cantidad de producto ofrecido, identificar variables de mercado y origen de los productores, la estacionalidad y las fluctuaciones en la oferta, la demanda y los precios que los comercializadores de las ferias del agricultor utilizan para estimar la oferta y demanda de los 20 productos agrícolas más comercializados en el mercado mayorista del CENADA.

Se realizaron en promedio 30 encuestas en cada feria del agricultor, para un total de 427, distribuidas como se muestra en el cuadro 4. Se observa que en ferias como las de Heredia, Desamparados y San Ramón se realizó la mayor cantidad de encuestas, debido a su tamaño. También se detalla como Zapote, Hatillo y Pavas tienen la menor cantidad de encuestas realizadas, lo cual es congruente con la cantidad de productores participantes en las ferias en estos lugares.

Cuadro 4. Distribución absoluta y relativa de las encuestas realizadas en cada feria del agricultor.

\begin{tabular}{|c|c|c|}
\hline \multirow{2}{*}{ Feria } & \multicolumn{2}{c|}{ Encuestas realizadas } \\
Cantidad & $\%$ \\
\hline Alajuelita & 29 & 6,8 \\
\hline Coronado & 37 & 8,7 \\
\hline Desamparados & 38 & 8,9 \\
\hline Escazú & 28 & 6,6 \\
\hline Grecia & 24 & 5,6 \\
\hline Hatillo & 21 & 4,9 \\
\hline Heredia & 46 & 10,8 \\
\hline Oreamuno & 36 & 8,4 \\
\hline Pavas & 22 & 5,1 \\
\hline Plaza Víquez & 30 & 7,0 \\
\hline San Ramón & 38 & 8,9 \\
\hline Tibás & 30 & 7,0 \\
\hline Tres Ríos & 28 & 6,6 \\
\hline Zapote & 20 & 4,7 \\
\hline Total & 427 & 100,0 \\
\hline
\end{tabular}

Fuente: Elaboración propia con datos de campo. 
Las encuestas se realizaron en dos periodos distintos; en 2013 se visitaron entre mayo y noviembre un total de ocho ferias del agricultor. En el año 2014, también entre mayo y noviembre, se visitaron las restantes seis ferias del agricultor que se estimaron en el cálculo de la muestra. El equipo de trabajo lo conformaron dos investigadores, dos asistentes y cinco encuestadores.

\section{Resultados y discusión}

Los entrevistados son comercializadores de productos agrícolas que tienen un puesto en alguna de las ferias del agricultor ubicadas dentro de la GAM, en adelante identificados como "ferieros". Existe una tendencia a incorporar a diferentes miembros de la familia en esta actividad, por lo que es habitual encontrar dueños o dueñas de fincas, esposas, esposos, hijos o hijas y otros parientes cercanos como encargados de la comercialización. Se entrevistaron 355 hombres, que corresponden al 85\% de la muestra, y 63 mujeres, que representan el 15\%.

Como se observa en la figura 1, del total de entrevistados, el 95\% asiste semanalmente a una feria, el $4 \%$ asiste a dos ferias y solamente el 1\% va a más de dos. El $71 \%$ de los ferieros encuestados mencionaron que tienen su plantación en el mismo lugar de residencia.

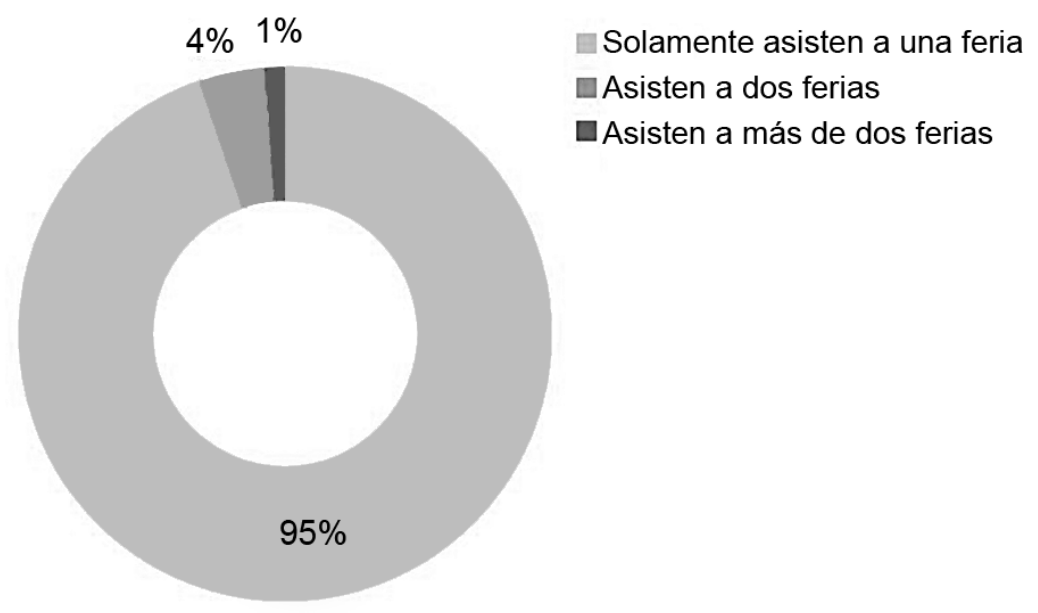

Figura 1. Entrevistados según la cantidad de ferias a las que asisten. Fuente: Elaboración propia con datos de campo.

En la Ley número 8533 sobre la regulación de las ferias del agricultor, en el artículo 33 se menciona lo siguiente: "si la producción lo amerita, podrán extenderse hasta dos carnés de vendedor auxiliar por finca, que los faculten para participar en un solo espacio por feria; sin embargo, podrán asistir a diferentes ferias" (CNP, 2008). Lo anterior es congruente con los datos que se observan en la figura 1 , donde un $4 \%$ de los entrevistados mencionan participar en más de una feria, lo cual está ligado al tamaño de la actividad productiva y justifica su participación en otras ferias.

Del total de los ferieros, el 43\% aseguró ser agricultor y comercializador de los productos que lleva a la feria. Esto se explica porque mantienen un sistema de organización según el cual, además de traer los productos que ellos mismos cosechan, comercializan productos de otros agricultores de la zona. Un 57\% se identificó únicamente como agricultor.

Tal y como se muestra en el cuadro 5, de aquellos que dijeron ser solo agricultores, prácticamente más de la mitad ha comprado algún producto agrícola a otro productor y el 46,3\% 
asegura nunca haberlo hecho. En este sentido, queda de manifiesto la falta de claridad de los agricultores sobre la definición o características de la intermediación, esto queda demostrado con los datos que aparecen en el cuadro citado, en el cual el 53,8\% ha realizado algún tipo de intermediación, sin embargo, no se consideran a sí mismos intermediarios.

Esta misma falta de claridad está presente en los agricultores-intermediarios; en este caso, un $74,3 \%$ indica que en algún momento ha comprado productos a otros productores, sin embargo, el 25,7\% afirma no hacerlo. Esto se explica por varios motivos, entre ellos la participación de la agricultura familiar en estos mercados, en los que el vínculo familiar contribuye a aumentar la oferta de productos del feriero; otro motivo es la participación de grupos de productores organizados formal o informalmente que tienen un único representante para la comercialización en la feria.

Cuadro 5. Tipo de feriero según la participación en la compra a otros productores.

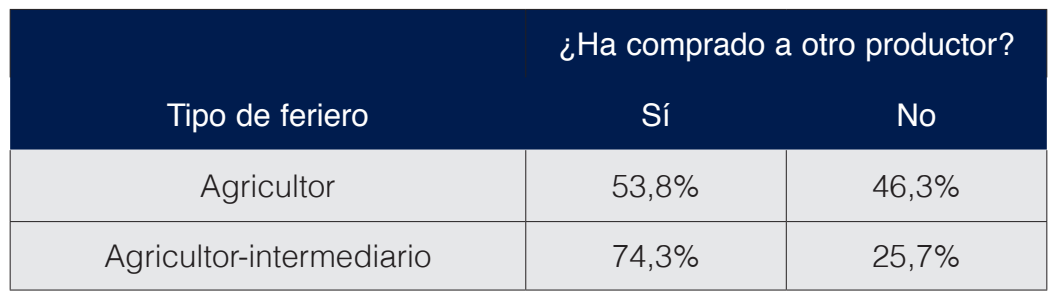

Fuente: Elaboración propia con datos de trabajo de campo.

Sin embargo, en la Ley 8533 sobre la regulación de las ferias del agricultor, capítulo uno, artículo primero, se decretan las disposiciones generales: "créase el Programa Nacional de Ferias del Agricultor, como programa de mercadeo de carácter social, de uso exclusivo para los pequeños y medianos productores nacionales de los sectores de la producción agrícola, pecuaria y forestal, pesca y acuicultura, avicultura, agroindustria y artesanía, en forma individual u organizada con el objeto de poner en relación directa a consumidores y productores, de manera tal que los primeros obtengan mejor precio y calidad, y los segundos incrementen su rentabilidad, al vender de modo directo al consumidor" (CNP, 2008).

En mercadotecnia, este tipo de plaza se conoce como canal de comercialización directo, o de nivel cero, lo que explica la relación directa entre productores y consumidores, en ausencia de algún tipo de intermediación, como se muestra en la figura 2, donde está claro que, según lo expuesto por la ley, se pretende que ambas partes obtengan un beneficio.

De acuerdo con la información obtenida mediante el estudio y según consta en el cuadro 4, sobre el perfil de los ferieros que participan en la muestra seleccionada, el 53,8\% de los agricultores y el $74,3 \%$ de los agricultores-intermediarios compran producto a otros productores. Esto plantea un esquema diferente de comercialización, donde aparece una intermediación, pasando de ser canal de comercialización directo a canal de comercialización indirecto nivel 1; por lo tanto, el nuevo esquema obedece a una mezcla entre lo que se dice canales de comercialización directos e indirectos, tal y como se muestra en la figura 3.

En mercadotecnia, y según lo expone Kotler, existe una diferencia entre los consumidores y los clientes. Los primeros son las personas que eventualmente realizan una compra, ya que andan en busca de satisfacción y bienestar a largo plazo, mientras que los segundos son aquellas personas que ya obtuvieron esa satisfacción y bienestar a largo plazo, por lo cual repiten 
la compra y crean en alguna medida un grado de lealtad hacia el producto, una marca y/o empresa, o ferieros en el caso de esta investigación (Kotler, 2012).

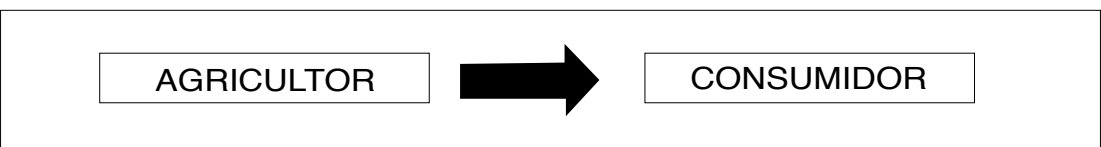

Figura 2. Canal de comercialización directo o de nivel cero, según la Ley 8533 de la República de Costa Rica.Fuente: Elaboración propia.

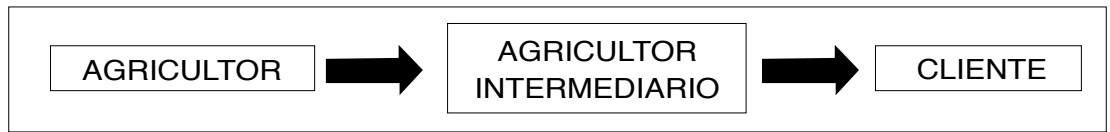

Figura 3. Canal de comercialización directo e indirecto. Fuente: Elaboración propia.

Se analizó, además, el comportamiento de la clientela de cada uno de los productos analizados en la investigación. Como se muestra en el cuadro 6, se pudo determinar que existe relación entre los consumidores y los productos de cada feriero, tal es el caso que, en promedio, el porcentaje de clientela habitual (lealtad de compra) oscila aproximadamente entre 40-60\% entre los distintos productos analizados. Se determinó que el producto con menor porcentaje de clientela habitual es la vainica, con un 41,3\%, mientras que el más alto corresponde a la piña, con un $58,2 \%$.

Cuadro 6. Porcentaje de clientela habitual según el producto.

\begin{tabular}{|c|c|c|c|}
\hline Producto & Porcentaje & Producto & Porcentaje \\
\hline Banano & 45,7 & Papaya & 57,4 \\
\hline Cebolla & 46,7 & Pepino & 47,4 \\
\hline Chayote & 42,6 & Piña & 58,2 \\
\hline Chile dulce & 50,1 & Plátano & 51,7 \\
\hline Coliflor & 55,7 & Repollo & 50,9 \\
\hline Limón & 46,3 & Sandía & 57,7 \\
\hline Manga & 47,9 & Tomate & 46,6 \\
\hline Melón & 50,0 & Vainica & 41,3 \\
\hline Naranja & 45,3 & Yuca & 51,2 \\
\hline Papa & 52,6 & Zanahoria & 57,0 \\
\hline
\end{tabular}

Fuente: Elaboración propia con datos de trabajo de campo.

Lo analizado anteriormente viene a complementarse con la información del cuadro 7, que muestra que para el caso específico de la feria de Tibás, los ferieros tienen una media de 74,6\% de clientela habitual, siendo esta la feria de más alto porcentaje. Por el contrario, en la feria de Zapote el promedio es de 29,7\%. 
Cuadro 7. Porcentaje de clientela habitual por feria.

\begin{tabular}{|c|c|c|c|}
\hline \multicolumn{1}{|c|}{ Feria } & Porcentaje & Feria & Porcentaje \\
\hline Grecia & 54,7 & Zapote & 29,7 \\
\hline San Ramón & 48,2 & Desamparados & 34,3 \\
\hline Coronado & 41,5 & Alajuelita & 45,2 \\
\hline Hatillo & 37,1 & Plaza Víquez & 41,0 \\
\hline Escazú & 59,1 & Oreamuno & 58,7 \\
\hline Tibás & 74,6 & Tres Ríos & 47,4 \\
\hline Pavas & 52,8 & Heredia & 49,6 \\
\hline
\end{tabular}

Fuente: Elaboración propia con datos de trabajo de campo.

En el caso de la regularidad de la oferta, se pudo determinar que un mismo comercializador normalmente tenía, al momento de ser entrevistado, más de un producto de los contemplados en el estudio. Por lo tanto, no es posible generalizar y hablar de una proporción de entrevistados que ofertan la misma cantidad de producto semanalmente en la feria durante todo el año. Sin embargo, sí se calculó la proporción para cada producto por tramo analizado, tal y como se puede observar en el cuadro 8.

La sandía y la manga obtuvieron los porcentajes más bajos de puestos con oferta constante, $25 \%$ y $38 \%$, respectivamente. Esto quiere decir que la oferta de estos productos oscila durante el año, debido principalmente a su estacionalidad, como se mencionó anteriormente. Es importante señalar que estos resultados pudieron verse afectados, además, por las fechas en las que se realizó el estudio, puesto que entre diciembre y febrero no se hicieron encuestas.

Por su parte, el pepino y la yuca son los productos con porcentajes de oferta constante más altos, $87 \%$ y $86 \%$, respectivamente; lo que significa que los ferieros tienden a mantener constante la cantidad que comercializan de estos dos productos semanalmente y durante el año.

De lo anterior, es importante mencionar que, del total de los 20 productos analizados, 18 mantienen más de un 50\% de la oferta constante a lo largo del año, lo que se justifica con el hecho de que existe un porcentaje de entre $40-60 \%$ de clientela fija por producto, lo que permite a los ferieros planificar o controlar de forma más efectiva las cantidades de productos que deben ofrecer semanalmente.

Solo un $6 \%$ de los ferieros menciona haber adquirido en algún momento producto de lotes destinados a la exportación para llevar a la feria. Los productos que se mencionaron son piña, yuca y manga.

De acuerdo con los datos de la figura 4, se observa la existencia del porcentaje total de ventas por tramo, que mantiene una alta rotación de productos, ya que cerca del $73 \%$ de los ferieros expresaron tener un porcentaje de ventas que varía entre $80-100 \%$ del total de producto que ofrecen semanalmente en las ferias en que participan, con una media del $96 \%$, lo que se considera alto para este tipo de mercado. Esto viene a reforzar la importancia de que los ferieros mantengan una cantidad mayor al $50 \%$ de clientela fija, que permite a los agricultores programar la oferta de productos, con cantidades más adaptadas a sus realidades y conocer épocas de mayor o menor demanda o la estacionalidad de ésta. Todo lo anterior genera resultados de mayores ventas y menores pérdidas y, por ende, mayor eficiencia en el proceso de comercialización. 
Cuadro 8. Porcentajes de puestos que mantienen constante la cantidad de productos que comercializan.

\begin{tabular}{|c|c|c|c|}
\hline Producto & $\%$ & Producto & $\%$ \\
\hline Banano & 71 & Papaya & 69 \\
\hline Cebolla & 65 & Pepino & 87 \\
\hline Chayote & 77 & Piña & 78 \\
\hline Chile dulce & 78 & Plátano & 68 \\
\hline Coliflor & 58 & Repollo & 77 \\
\hline Limón & 70 & Sandía & 25 \\
\hline Manga & 38 & Tomate & 77 \\
\hline Melón & 50 & Vainica & 62 \\
\hline Naranja & 70 & Yuca & 86 \\
\hline Papa & 75 & Zanahoria & 76 \\
\hline
\end{tabular}

Fuente: Elaboración propia con datos de trabajo de campo.

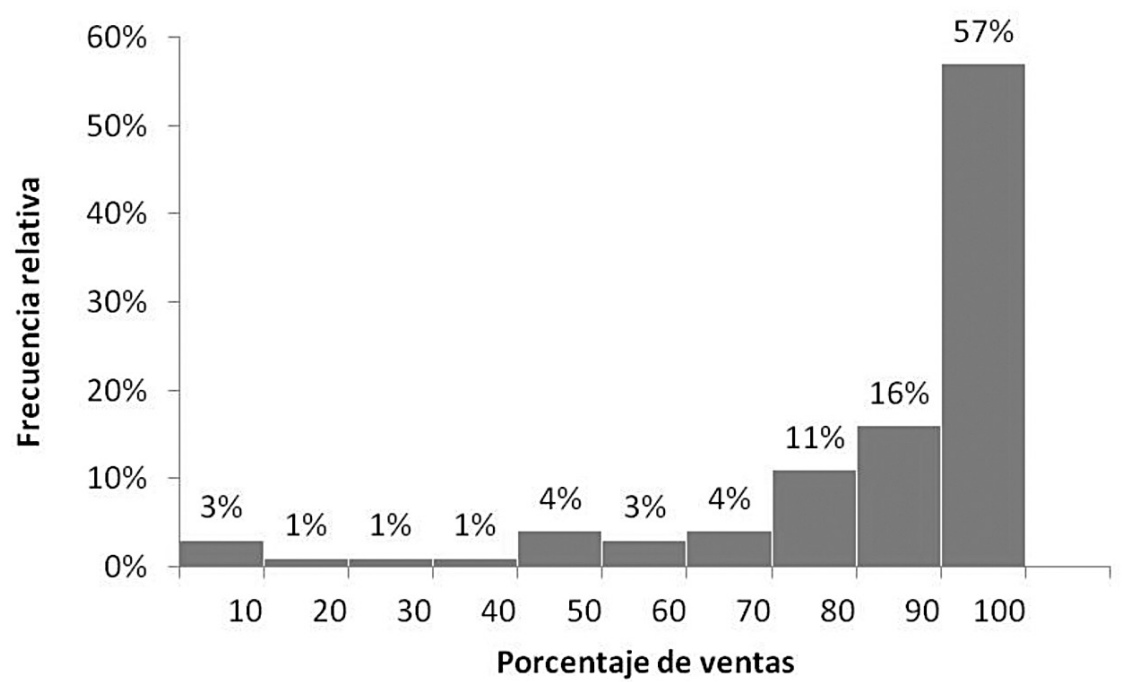

Figura 4. Distribución de los puestos según el porcentaje de producto vendido por semana en la feria. Fuente: Elaboración propia con datos de trabajo de campo.

Entre los 20 productos analizados no existen diferencias estadísticamente significativas en relación con este porcentaje de ventas. Sin embargo, entre las ferias incluidas en el estudio sí se encontrarón algunas diferencias; según el análisis de varianza y la prueba de LSD al 5\% de significancia, se puede ver en el cuadro 9 que la feria de Alajuelita tiene el promedio más bajo de ventas (84,76\%), lo que es estadísticamente diferente a las de Heredia, Desamparados, Grecia, Tibás y Pavas, que presentan los promedios más altos.

Es importante rescatar de este analisis que existe un porcentaje bastante alto de ventas en todas las ferias analizadas, superior al $84 \%$, eso indica que los productores planifican muy bien las cantidades de productos que suelen llevar semanalmente y durante todo el año a las ferias del agricultor. 
Cuadro 9. Análisis de varianza y prueba LSD al 5\% del porcentaje de ventas por feria.

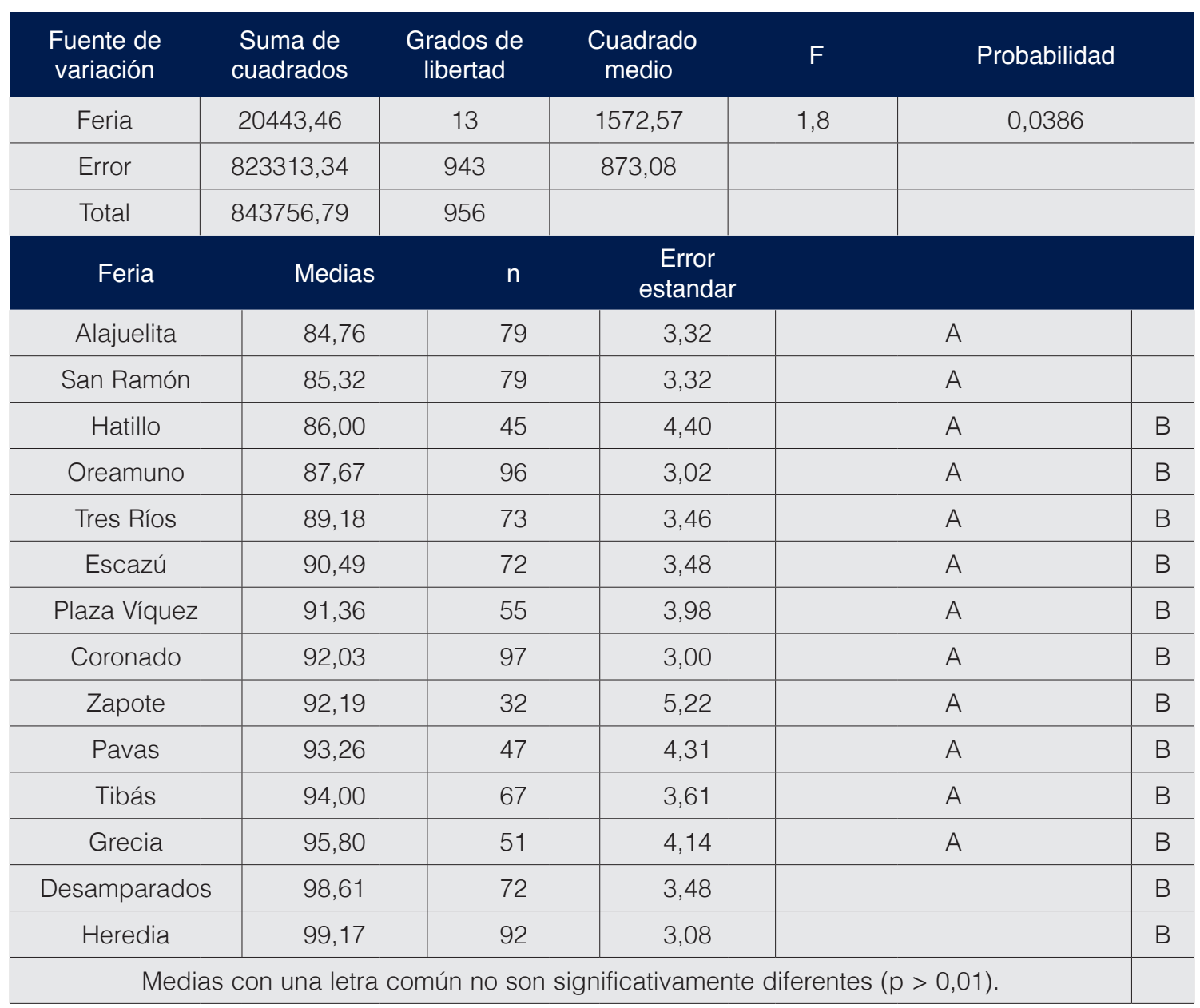

Fuente: Elaboración propia con datos de trabajo de campo.

Finalmente, la investigación determinó que apenas un 7\% de los ferieros entrevistados indican que tienen algún tipo de certificación, sin embargo, esta no corresponde a certificaciones de calidad de los productos o procesos productivos. Más bien, ellos hacen alusión, entre otros, a aspectos tales como tener el carné de manipulación de alimentos o el que los certifica como comercializadores de la feria del agricultor y que es expedido por el CNP, como se indica en su artículo "Entes administradores y emisores de carné de ferias del agricultor" (CNP, 2014a).

\section{Conclusiones}

De los productores que asisten a las diferentes ferias del agricultor analizadas se concluye que más del 90\% visitan cada semana únicamente una. Este es un dato bastante alto y refleja un comportamiento estable, a pesar de que la legislación les permite participar en más de una feria.

El 57\% de la población encuestada afirmó ser agricultor, sin embargo, el 53,8\% de los que se catalogan como tales mencionan haber realizado compras de productos agrícolas para traer a las ferias, situación que está relacionada con la falta de claridad de los agricultores sobre la definición o características de la intermediación. 
El $43 \%$ de los encuestados se relaciona con agricultores-intermediarios; el $74,3 \%$ de estos menciona haber comprado producto para llevar a las ferias, lo que se justifica por cuanto ellos mismos se han reconocido como agricultores-intermediarios, sin embargo, el $25,7 \%$ de estos menciona no haber realizado nunca compra de productos agrícolas a otros productores. En este caso, de nuevo se manifiesta la falta de claridad sobre el concepto de intermediación, en el que está presente la participación de la agricultura familiar para aumentar la oferta de productos del feriero; y otro motivo es la participación de grupos de productores organizados formal o informalmente por uno de ellos.

Se determinó que los ferieros cuentan con clientela fija para cada uno de los productos analizados; si bien los porcentajes varían de acuerdo con los productos, la media está entre 40-60\%. También se determinó que de una feria a otra la clientela habitual puede variar, siendo la de Tibás la que presenta el promedio más alto y la de Zapote el más bajo.

A pesar de que la Ley 8533 establece que los participantes en las ferias serán productores (agricultores), se identificó un tipo de intermediación que crea una mezcla de agricultorintermediario. Sus características son ser productor agrícola y a la vez, para poder abastecer las necesidades de sus clientes fijos, en eventuales ocasiones en las que carece de productos para cumplir con la demanda, se ve obligado a adquirir producto de otros agricultores y así cumplir con el principio básico de la mercadotecnia, que es satisfacer las necesidades del segmento al que se dirige.

De los 20 productos analizados, que corresponden a los de mayor consumo en el mercado mayorista del CENADA, 18 mantienen un porcentaje que varía entre 50-83\% de oferta constante a lo largo del año. Solo la sandía y el mango están por debajo de estos porcentajes y su comportamiento se explica por el carácter estacional que presentan ambas frutas.

En relación con el comportamiento de los porcentajes de ventas, el 80\% de la población analizada asegura que logra vender entre $84-100 \%$ de la oferta que lleva cada semana a las ferias. Esto permite constatar que los ferieros planifican eficientemente la cantidad de producto que ofrecen, algo que, sin duda alguna, está estrechamente relacionado con los porcentajes tan altos de clientes fijos.

\section{Bibliografía}

Arellano, R. (2000). Marketing: enfoque América Latina. 1 ed. México: McGraw Hill.

Benassini, M. (2001). Introducción a la investigación de mercados: un enfoque para América Latina. México: Pearson Educación.

CNP (Consejo Nacional de Producción). (18 septiembre, 2008). Reglamento a la Ley de Regulación de Ferias del Agricultor. La Gaceta No 180. Disponible en http://www.cnp.go.cr/ferias/leyes/Reglamento_Ley_8533.pdf.

CNP (Consejo Nacional de Producción) (2014a). Entes administradores y emisores de carné de ferias del agricultor. Disponible en http://www.cnp.go.cr/ferias/listas/Lista_Ferias_Agricultor.pdf.

CNP (Consejo Nacional de Producción). (2014b). ¿Qué son las ferias? Disponible en http://www.cnp.go.cr/ferias/

Hair, J., Bush, R. \& Ortinau, D. (2010). Investigación de mercados: en un ambiente de información digital. 4 ed. México: Mc Graw Hill.

Hoyer, W. \& Maclnnis, D. (2010). Comportamiento del Consumidor. 5 ed. México: Cengage Learning.

Kotler, P. \& Armstrong, G. (2012). Marketing. 14 ed. México: Pearson Educación.

Little, T. \& Hills, F. (1983). Statistical methods in agricultural research. México: Editorial Trillas, S.A.

Ministerio de Agricultura y Ganadería. (2006). Regulación de Ferias del Agricultor. Disponible en http://www.mag. go.cr/legislación/2006/ley-8533.pdf 
Ministerio de Vivienda y Asentamientos Humanos (2011). Plan de ordenamiento territorial de la Gran Área Metropolitana 2011-2030. Disponible en http://www.mivah.go.cr/Documentos/potgam/PROPUESTAS 3_21_ AGOSTO_2012.pdf

Montgomery, D. (2004). Diseño y análisis de experimentos. 1 ed. México: Limusa S.A.

Navarro, J.R. (2006). Diseño experimental: aplicaciones en agricultura. 1 ed. Costa Rica: Editorial Universidad de Costa Rica.

Zikmund, W. \& Babin, B. (2009). Investigación de mercados. 9 ed. México: Cengage Learning. 